\title{
Fluorometrische Untersuchungen über die Bildung von Enzym-Coenzym-Komplexen der Lactat-Dehydrogenase aus Schweineherz mit Coenzymbruchstücken und Coenzymmodellen
}

\author{
Fluorometric Investigation of the Formation of Enzyme-Coenzyme-Complexes \\ of Pig Heart Lactate Dehydrogenase with Coenzyme Fragments and Coenzyme Analogs \\ C. Woenckhaus und R. JeCK \\ Institut für Vegetative Physiologie, Chemisch-Physiologisches Institut, Abteilung für Enzymologie \\ der J. W. Goethe-Universität, Frankfurt am Main \\ (Z. Naturforschg. 24 b, 1436-1441 [1969] ; eingegangen am 6. Mai 1969)
}

\begin{abstract}
Fluorescense spectra of lactate dehydrogenase * (E.C. 1.1.1.27) were investigated in the presence of the coenzyme fragments dihydronicotinamide mononucleotide and dihydronicotinamide-ribose- $5^{\prime}$. pyrophospho- $\left(\mathrm{P}^{2}\right)-5^{\prime \prime}$-ribose. The reduced mononucleotide is enzymatically less active as a hydrogen donor. However, formation of a complex with the enzyme was not observed under the conditions used. All the other substances: dihydronicotinamide-ribose-5'-pyrophospho- $\left(\mathrm{P}^{2}\right)-5^{\prime \prime}$-ribose, dihydronicotinamide- benzimidazole-dinucleotide, dihydronicotinamide-3-desazapurine-dinucleotide and dihydronicotinamide-6-mercaptopurine-dinucleotide form more or less stable complexes with lactate dehydrogenase. The complexes do not markedly differ from the complex formed with the natural cofactor. In all cases spectra indicate change in conformation of the coenzyme by forming the coenzyme-enzyme-complex which has been proposed by VELICK ${ }^{1}$ too. The cysteine residues of the lactate dehydrogenase are not essential for binding the coenzyme to the active center; this was shown with mercury blocked enzyme.
\end{abstract}

Lactat-Dehydrogenase zeigt in Pufferlösungen nach Einstrahlen von Licht der Wellenlänge $288 \mathrm{~m} \mu$ eine charakteristische Fluoreszenzemission mit einem Maximum bei $340 \mathrm{~m} \mu$. Diese Eiweißfluoreszenz wird durch Zugabe von Adenosindiphosphat, Adenosindiphosphat-ribose und Nicotinamid-adenin-dinucleotid unterdrückt. Besonders mit Dihydronicotinamid-adenin-dinucleotid * bildet das Enzym einen wenig dissoziierten Komplex. Das Fluoreszenzanregungs- und -emissionsspektrum des Coenzym-Enzym-Komplexes wurde von VELICK ${ }^{1}$, KAPLAN und Mitarbb. ${ }^{2}$ und WINER und SCHWERT ${ }^{3}$ eingehend untersucht.

Die Fluoreszenzemission des DihydrocoenzymEnzym-Komplexes nach Einstrahlen von Licht der Wellenlänge $300 \mathrm{~m} \mu$ setzte sich nicht additiv aus den Fluoreszenzintensitäten der Einzelkomponenten: Enzym und Dihydrocoenzym zusammen, sondern zeigte ab $410 \mathrm{~m} \mu$ in Richtung zum langwelligen Bereich eine Intensitätszunahme und in Richtung zu

* Abkürzungen: LDH = Lactat Dehydrogenase, L-Lactat: NAD-Oxido-reduktase (E.C. 1.1.1.27); NAD = Nicotinamid-adenin-dinucleotid; $\mathrm{NADH}=$ Dihydronicotinamidadenin-dinucleotid.

1 S. F. Velick, J. biol. Chemistry 233, 1455 [1958].

2 S. Shifrin, N. O. Kaplan u. M. M. Ciotti, J. biol. Chemistry 234, 1555 [1958]. kürzeren Wellenlängen eine Unterdrückung der Fluoreszenz. Nach VELICK ${ }^{1}$ wird das eingestrahlte Licht von Tryptophanresten des Enzyms auf den Dihydronicotinamidring übertragen und als Fluoreszenzlicht von ca. $460 \mathrm{~m} \mu$ emittiert. Auf Grund der fehlenden Intensitätszunahme im Absorptionsbereich des Adeninteils bei $260 \mathrm{~m} \mu$ soll das Coenzym in einer anderen Konformation als in Lösung an das aktive Zentrum des Enzyms gebunden werden. SHIFRIN ${ }^{4}$ zeigte durch das optische Verhalten von $N$ - ( $\beta$-Indolyläthyl) -3-carbamoyl-dihydropyridin, daß der funktionelle Coenzymteil einem Tryptophanrest benachbart sein kann. Bei unseren Untersuchungen über die Hemmung der LDH mit 3-Bromacetylpyridin und nach tryptischer Spaltung des denaturierten Enzyms fanden wir ein Peptid, in dem neben der modifizierten Aminosäure Histidin noch ein Tryptophanrest enthalten war ${ }^{5}$. Die Coenzymteile, Adenosindiphosphat, Adenosindiphosphat-ribose und NAD, verhindern den Angriff von 3-Bromacetylpyridin an

\footnotetext{
3 A. D. Winer, G. W. Schwert u. D. B. S. Millar, J. biol. Chemistry 234, 1149 [1958].

4 S. Shifrin, Biochim. biophysica Acta [Amsterdam] 81 . 205 [1964].

5 C. Woenckhaus, J. Berghäuser u. G. Pfleiderer, Hoppe-Seyler's Z. physiol. Chem. 350, 473 [1969].
} 
das Enzym; Nicotinamidmononucleotid hatte dagegen keinen Schutzeffekt. Wir vermuteten daher, daß der Hemmstoff eine kovalente Bindung an der Haftstelle des Coenzym-adeninteils bewirkt. Durch fluorometrische Untersuchungen mit den Coenzymbruchstücken Dihydronicotinamidmononucleotid und Dihydronicotinamid-ribose- $5^{\prime}$-diphospho- $\left(\mathrm{P}^{2}\right)-5^{\prime \prime}$-ribose und mit den Coenzymanalogen Dihydronicotinamid-benzimidazoldinucleotid, Dihydronicotinamid3-desazapurin-dinucleotid und Dihydronicotinamid6-mercaptopurin-dinucleotid versuchten wir Aussagen über die Bindung des nichtfunktionellen Molekülteils an das Enzym zu machen.

\section{Material und Methoden}

Enzyme und Coenzyme: Lactat-Dehydrogenase aus Schweineherz (E.C. 1.1.1.27), Alkohol-Dehydrogenase aus Hefe (E.C. 1.1.1.1), NADH, NAD, Nicotinamidmononucleotid, Adenosindiphosphat-ribose wurden von der Firma Boehringer \& Soehne bezogen.

Die Darstellung der Coenzymmodelle: Nicotinamidribose-5'-diphospho- $\left(\mathrm{P}^{2}\right)-5^{\prime \prime}$-ribose ${ }^{6}$, Nicotinamid-benzimidazol-dinucleotid ${ }^{7}$, Nicotinamid-3-desazapurin-dinucleotid $^{8,14}$ und Nicotinamid-6-mercaptopurin-dinucleotid ${ }^{9}$ haben wir an anderer Stelle beschrieben.
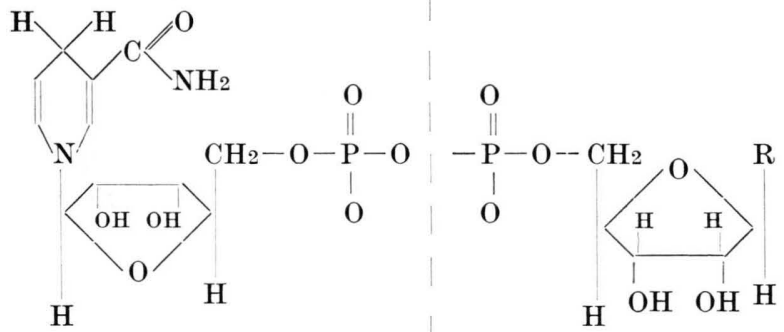

1. Dihydronicotinamid-mononucleotid.

2. $\mathrm{R}=\mathrm{OH}$; Dihydronicotinamid-ribose-5' - diphospho$\left(\mathrm{P}^{2}\right)-5^{\prime \prime}$-ribose.
3. $\mathrm{R}$<smiles>c1csc2ncnc-2c1</smiles>

4. $\mathrm{R}=$<smiles>c1=c2nccc-2-s-c2nc-1n2</smiles>

Dihydronicotinamidbenzimidazol-dinucleotid.

Dihydronicotinamid-3desazapurindinucleotid.
6 C. Woenckhaus u. M. Volz, Chem. Ber. 99, 1712 [1966].

7 C. Woenckhaus, Chem. Ber. 97, 2439 [1964].

8 C. Woenckhaus u. G. Pfleiderer, Biochem. Z. 341, 495 [1965].

9 G. Pfleiderer, C. Woenckhaus, K. Scholz u. H. Feller, Liebigs Ann. Chem. 675, 205 [1964].
5. $\mathrm{R}=\underline{\mathrm{N}}_{\overline{\mathrm{N}}}^{\overline{\mathrm{S}} \mathrm{H}}$

Dihydronicotinamid-6mercaptopurindinucleotid.

Quecksilberblockierte Lactat-Dehydrogenase: Sie wurde analog einer Vorschrift von PfLeiderer und JECKEL ${ }^{10}$ hergestellt.

Reduktion der Coenzymmodelle: $75 \mathrm{mg}$ des Coenzymanalogen wurde in $5 \mathrm{ml} 0,2 \mathrm{M}$ Glycin/ $\mathrm{NaOH}$-Puffer pH 9,5 gelöst. Der pH-Wert wurde mit verdünnter $\mathrm{Na}$ tronlauge nachgestellt, sodann der Ansatz mit $0,5 \mathrm{ml}$ Äthanol und $0,05 \mathrm{ml}$ einer Alkohol DehydrogenaseSuspension $(30 \mathrm{mg} / \mathrm{ml})$ versetzt. Nach $30 \mathrm{Min}$. wurde bei einer 1:100 verdünnten Probe keine weitere $\mathrm{Zu}$ nahme der Extinktion bei $340 \mathrm{~m} \mu$ festgestellt. Die Abtrennung des Coenzyms aus dem Reaktionsgemisch erfolgte an einer Sephadex-G 25 fine-Säule $(100 \cdot 2 \mathrm{~cm})$ mit 0,2 M Glycin/Natronlauge-Puffer $\mathrm{pH}$ 9,5. Konzentration und Reinheit der eluierten Coenzyme wurden jeweils durch Aufnahme der UV-Spektren bestimmt.

Dihydronicotinamid-mononucleotid und Dihydronicotinamid-ribose-5'-diphospho- $\left(P^{2}\right)-5^{\prime \prime}$-ribose: Sie wurden nach einer modifizierten Methode von STEWART und Mitarbb. ${ }^{11,12}$ mit Natriumdithionit hergestellt.

Die Abtrennung von Verunreinigungen, Salzen und der oxydierten Verbindung erfolgte an einer SephadexG 10-Säule $(150 \cdot 1,5 \mathrm{~cm})$ durch Elution mit 0,1-proz. Ammoniumhydrogencarbonat/Ammoniak-Puffer pH 8. Dihydronicotinamid-ribose- $5^{\prime}$-diphospho - $\left(\mathrm{P}^{2}\right)-5^{\prime \prime}$ - ribose zersetzte sich in einigen Stdn. unter Bildung des primären Säureprodukts.

Lactat-Dehydrogenase: $2 \mathrm{ml}$ Kristallsuspension 10 $\mathrm{mg} / \mathrm{ml}$ wurden zentrifugiert, der Niederschlag in $0,5 \mathrm{ml}$ $0,2 \mathrm{M}$ Glycin/Natronlauge-Puffer $\mathrm{pH}$ 9,5 aufgenommen und durch Chromatographie an der Sephadex-G 25 fineSäule $(100 \cdot 2 \mathrm{~cm})$ von Salzen befreit. Die Enzymkonzentration wurde durch die Biuretmethode mit dem Faktor 16,5 bestimmt. Die Enzymkonzentration in der Küvette betrug $0,36 \mathrm{mg} / \mathrm{ml}\left(1 \cdot 10^{-5} \mathrm{M}\right)$, bezogen auf die Untereinheit LDH Mol.-Gew. 36000 . Die Coenzymkonzentrationen sind in den Legenden der Abbildungen angegeben. Alle Messungen wurden in 0,2 M Glycin/ Natronlauge-Puffer $\mathrm{pH}$ 9,5 durchgeführt.

Die Bestimmung der Dissoziationskonstanten der Coenzym-Enzym-Komplexe erfolgte nach der Vorschrift von VELICK ${ }^{1}$.

Die Absorptionsspektren wurden im Zeiss PMQII Spektralphotometer aufgenommen. Zur Messung der Fluoreszenzanregungs- und -emissionsspektren verwandten wir ein Beckman Gitterfluorometer mit einer $450 \mathrm{~W}$ att Xenon-Lampe. Die Temperatur betrug $25^{\circ} \mathrm{C}$.

Enzymatische Oxydation des Dihydronicotinamid-

10 G. Pfleiderer u. D. Jeckel, Europ. J. Biochem. 2, 171 [1967].

11 G. Pfleiderer, C. Woenckhaus u. M. Nelböck-HochStetTer, Liebigs Ann. Chem. 690, 170 [1965].

12 S. Gutcho u. E. D. Stewart, Analytic. Chem. 20, 1185 [1948]. 
mononucleotids: In einer Kuvette $D=1 \mathrm{~cm}$ befanden sich in $3 \mathrm{ml} 0,1 \mathrm{M}$ Tris/HCl-Puffer $\mathrm{pH} 7,0,0,1 \mathrm{mMol}$ Pyruvat und 0,2 $\mu \mathrm{Mol}$ Dihydronicotinamid-mononucleotid. Die Reaktion wurde mit 1,0 mg LDH ausgelöst und die Abnahme der charakteristischen Fluoreszenz bei $460 \mathrm{~m} \mu$ und die der Absorption bei $340 \mathrm{~m} \mu$ bei $22^{\circ}$ gemessen. Blindwerte, denen das Enzym oder das Pyruvat nicht zugesetzt war, zeigten innerhalb einer Stde. nur eine geringe Verminderung der Extinktion oder Fluoreszenz.

\section{Ergebnisse}

Dihydronicotinamid-mononucleotid ist im Test mit LDH als Wasserstoffüberträger schwach wirksam. Etwa 50\% des eingesetzten Coenzymbruchstückes wurden in Gegenwart von 0,1 м Pyruvat bei pH 7 innerhalb von $30 \mathrm{Min}$. durch $\mathrm{LDH}$ oxidiert. Die größere Aktivität der hydrierten Coenzymbruchstücke gegenüber den oxidierten wurde von SIEBERT $^{15}$ an der unspezifischen Alkohol-Dehydrogenase aus Leber beobachtet. Im Fluoreszenzanregungs- und -emissionsspektrum konnten wir auch nach Zugabe von Pyruvat keine Änderung außer einer langsamen Intensitätsabnahme feststellen. Die Proteinfluoreszenz nach Anregung bei $290 \mathrm{~m} \mu$ und die entsprechende Emission bei $340 \mathrm{~m} \mu$ wurde auch bei einem 50-fachen Überschuß gegenüber dem Enzym nicht verändert. Die Fluoreszenzemissionsund -anregungsspektren setzten sich additiv aus den beiden Komponenten LDH und Coenzymbruchstück zusammen (Abb. 1 und 2).

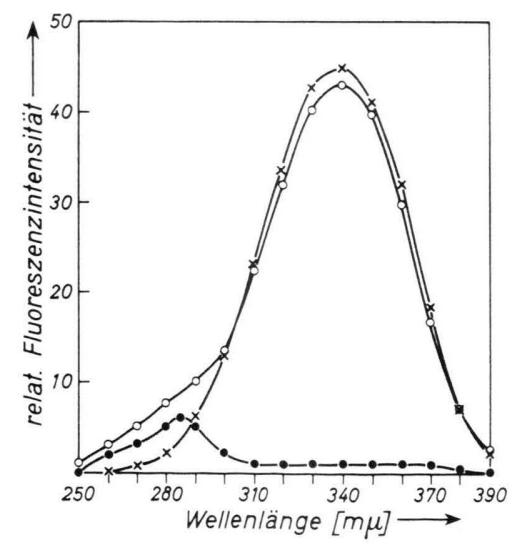

Abb. 1. Fluoreszenzanregungs-Spektrum: Emission $460 \mathrm{~m} \mu$. $x--x$ Dihydronicotinamidmononucleotid, $c=5 \cdot 10^{-4} \mathrm{M}$; $\bullet-\bullet \mathrm{LDH}, c=1 \cdot 10^{-5} \mathrm{M} ; 0--\mathrm{O}$ Dihydronicotinamidmononucleotid, $c=5 \cdot 10^{-4} \mathrm{M}$ und $\mathrm{LDH}, c=1 \cdot 10^{-5} \mathrm{M}$; gemessen in $0,2 \mathrm{M}$ Glycin/NaOH-Puffer $\mathrm{pH}$ 9,5. Empfängerempfindlichkeit: Stufe 7.

13 G. Weber, Nature [London] 180, 1409 [1957].

14 C. Woenckhaus u. P. Zumpe, Z. Naturforschg. 23 b, 484 [1968].

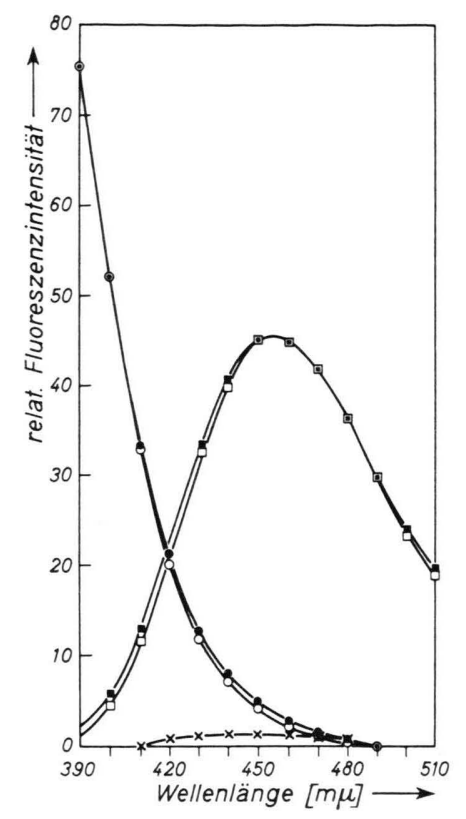

Abb. 2. Fluoreszenzemissions-Spektrum: Anregung $280 \mathrm{~m} \mu$. $\mathrm{O}-\mathrm{O} \mathrm{LDH}, c=1 \cdot 10^{-5} \mathrm{M} ;--\bullet \mathrm{LDH}, c=1 \cdot 10^{-5} \mathrm{M}$ und Dihydronicotinamidmononucleotid, $c=5 \cdot 10^{-4} \mathrm{M}$; Fluoreszenzemisions-Spektrum: Anregung $340 \mathrm{~m} \mu . \times--x$ LDH, $c=1 \cdot 10^{-5} \mathrm{M} ; \square--\square$ Dihydronicotinamidmononucleotid, $c=5 \cdot 10^{-4} \mathrm{M}$; - $\mathrm{LDH}, c=1 \cdot 10^{-5} \mathrm{M}$ und Dihydronicotinamidmononucleotid, $c=5 \cdot 10^{-4} \mathrm{M}$; gemessen in $0,2 \mathrm{M}$ Glycin/ $\mathrm{NaOH}$-Puffer $\mathrm{pH}$ 9,5. Empfängerempfindlichkeit: Stufe 7.

Die Fluoreszenzintensität des Dihydronicotinamid-mononucleotids bei $460 \mathrm{~m} \mu$ bei Anregung mit Licht von $340 \mathrm{~m} \mu$ ist etwas geringer als im intakten Coenzym. Dieser Befund wurde schon von WeBER ${ }^{13}$ beobachtet und liegt sehr wahrscheinlich im Auftreten einer zusätzlichen Deformationsschwingung des Mononucleotidteils nach Spaltung der Pyrophosphatbildung. Bei der Verwendung des Coenzymbruchstücks Dihydronicotinamid-ribose-5'. diphospho- $\left(\mathrm{P}^{2}\right)-5^{\prime \prime}$-ribose trat dagegen die Ausbildung eines Coenzym-Enzym-Komplexes ein. Dieser Komplex hatte eine Dissoziationskonstante $K=$ $18 \cdot 10^{-6} \mathrm{M}, \mathrm{pH} 9,5$. Sie ist etwa $60-\mathrm{mal}$ größer als die des NADH-LDH-Komplexes $K=0,30 \cdot 10^{-6} \mathrm{M}$. Die schwächere Bindung steht im Einklang mit der größeren $\mathrm{Mich}$ a elis-Konstanten ${ }^{14}$. Das Fluoreszenzemissions-Spektrum nach Anregung bei 280 $\mathrm{m} \mu$ (Abb. 3 Teil A) des Komplexes zeigte eine Intensitätsverminderung ab $410 \mathrm{~m} \mu$ zu kürzeren Wellenlängen, wie sie von VELICK ${ }^{1}$ für den NADHLDH-Komplex beschrieben wurde (Abb. 3 Teil B).

15 K. Kesselring u. G. Siebert, Hoppe-Seyler's Z. physiol. Chem. 337, 79 [1964]. 


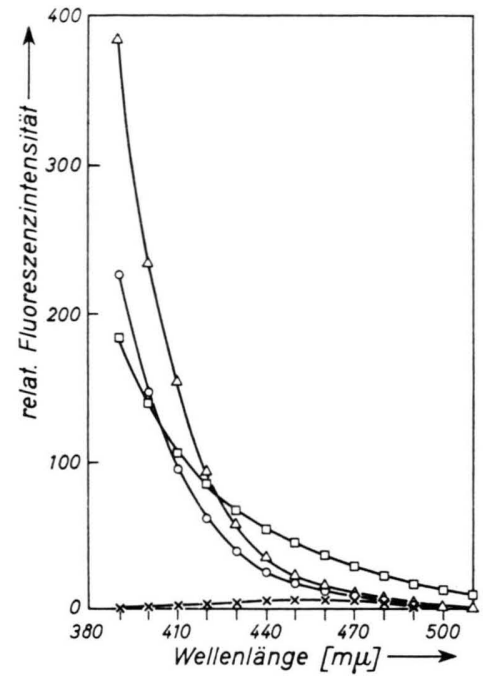

Abb. 3 A. Fluoreszenzemissions-Spektrum: Anregung $280 \mathrm{~m} \mu$. $\triangle-\triangle \triangle \mathrm{LDH}, c=1 \cdot 10^{-5} \mathrm{M} ; \mathrm{O}-0 \mathrm{OLDH}, c=1 \cdot 10^{-5} \mathrm{M}$ und Adenosindiphosphatribose $c=1 \cdot 10^{-4} \mathrm{M} ; \square--\square$ LDH, $c=1 \cdot 10^{-5} \mathrm{M}$ und Dihydronicotinamid-ribosid-5'-pyrophospho- $\left(\mathrm{P}^{2}\right)-5^{\prime \prime}$-ribose, $c=1 \cdot 10^{-4} \mathrm{M} ; \times--\times$ Dihydronicotinamid-ribosid-5'-pyrophospho- $\left(\mathrm{P}^{2}\right)-5^{\prime \prime}$-ribose, $c=1 \cdot 10^{-4} \mathrm{M}$; gemessen in $0,2 \mathrm{M}$ Glycin/NaOH-Puffer $\mathrm{pH}$ 9,5. Empfängerempfindlichkeit: Stufe 8.

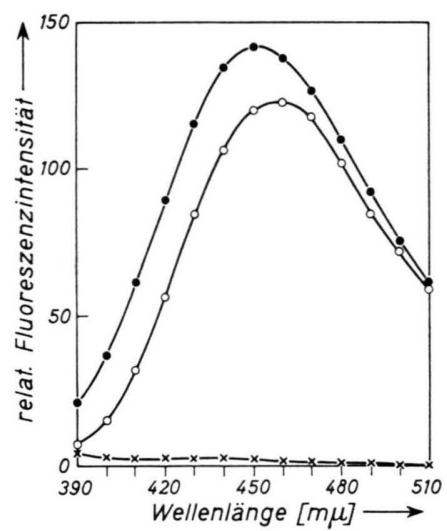

Abb. 3 B. Fluoreszenzemissions-Spektrum: Anregung $340 \mathrm{~m} \mu$. $\times--\times \mathrm{LDH}, c=1 \cdot 10^{-5} \mathrm{M} ; \mathrm{O}--\mathrm{O}$ Dihydronicotinamidribosid-5'-pyrophospho- $\left(\mathrm{P}^{2}\right)-5^{\prime \prime}$-ribose, $c=1 \cdot 10^{-4} \mathrm{M} ; \bullet--$ LDH, $c=1 \cdot 10^{-5} \mathrm{M}$ und Dihydronicotinamid-ribosid-5'-pyrophospho- $\left(\mathrm{P}^{2}\right)-5^{\prime \prime}$-ribose, $c=1 \cdot 10^{-4} \mathrm{M}$; gemessen in $0,2 \mathrm{M}$ Glycin/NaOH-Puffer $\mathrm{pH} 9,5$.

Mit einer gleichgroßen Konzentration Adenosindiphosphoribose erzielten wir eine ebenso starke Unterdrückung der Fluoreszenzemission.

Das Fluoreszenzanregungs-Spektrum von Dihyronicotinamidribose-5'-diphospho- $\left(\mathrm{P}^{2}\right)-5^{\prime \prime}$-ribose im Komplex mit LDH zeigte Steigerung der Fluoreszenzintensität sowohl im Anregungsbereich von 290 $\mathrm{m} \mu$ wie auch bei $340 \mathrm{~m} \mu$ (Abb. 4).

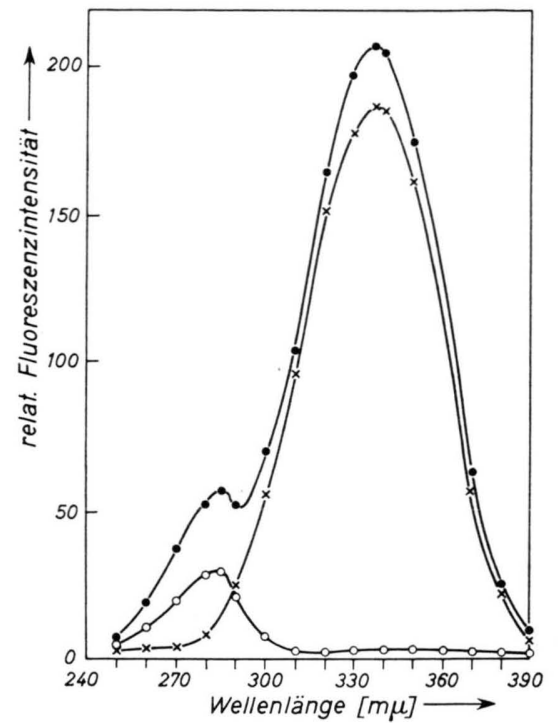

Abb. 4. Fluoreszenzanregungs-Spektrum: Emission $460 \mathrm{~m} \mu$. $x--x$ Dihydronícotinamid-ribosid-5'-pyrophospho- $\left(\mathrm{P}^{2}\right)-5^{\prime \prime}$ ribose, $c=1 \cdot 10^{-4} \mathrm{M} ; 0--0 \mathrm{LDH}, \quad c=1 \cdot 10^{-5} \mathrm{M}$; $\bullet--\mathrm{LDH}, c=1 \cdot 10^{-5} \mathrm{M}$ und Dihydronicotinamid-ribosid-5'-pyrophospho- $\left(\mathrm{P}^{2}\right)-5^{\prime \prime}$-ribose, $c=1 \cdot 10^{-4} \mathrm{M}$; gemessen in $0,2 \mathrm{M}$ Glycin/ $\mathrm{NaOH}$-Puffer $\mathrm{pH} 9,5$. Empfängerempfindlichkeit: Stufe 8.

Dihydronicotinamid-benzimidazol-dinucleotid bildete einen Komplex mit LDH, die Dissoziationskonstante betrug $K=6 \cdot 10^{-6} \mathrm{M}$. Im Fluoreszenzanregungs-Spektrum stellten wir nur einen geringen Unterschied in der Fluoreszenzintensität bei $460 \mathrm{~m} \mu$

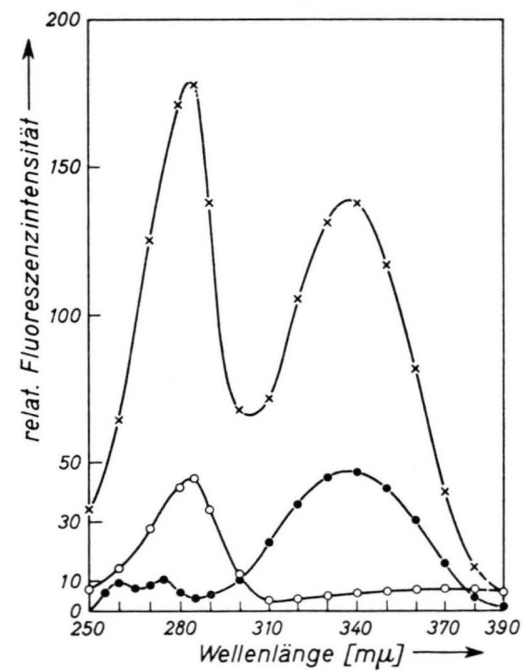

Abb. 5. Fluoreszenzanregungsspektrum: Emission $460 \mathrm{~m} \mu$. - - Dihydronicotinamid-benzimidazol-dinucleodid, $c=$ $1 \cdot 10^{-4} \mathrm{M} ; 0--\mathrm{LDH}, c=1 \cdot 10^{-5} \mathrm{M} ; \times--\times \mathrm{LDH}, c=$ $1 \cdot 10^{-5} \mathrm{M}$ und Dihydronicotinamid-benzimidazol-dinucleotid, $c=1 \cdot 10^{-4} \mathrm{M}$; gemessen in $0,2 \mathrm{M}$ Glycin/NaOH-Puffer $\mathrm{pH}$ 9,5. Empfängerempfindlichkeit: Stufe 8. 
gegenüber dem natürlichen NADH-LDH-Komplex fest. Das Doppelmaximum des freien Coenzymmodells im Fluoreszenzanregungs-Spektrum bei 260 und $275 \mathrm{~m} \mu$ wurde im Komplex mit dem Enzym nicht mehr beobachtet (Abb. 5).

Der Komplex aus Lactat Dehydrogenase und Dihydronicotinamid-3-desazapurin-dinucleotid weist eine Dissoziationskonstante von $K=2 \cdot 10^{-6} \mathrm{M}$ auf und ähnelt, wie der, den Dihydronicotinamid-6mercaptopurin-dinucleotid mit der LDH bildet, $K=$ $1.10^{-6} \mathrm{M}$, dem natürlichen NADH-LDH-Komplex. Im Dihydronicotinamid-6-mercaptopurin-dinucleotid absorbiert der 6-Mercaptopurinring bei $315 \mathrm{~m} \mu$. Eine Energieübertragung von dem 6-Mercaptopurin auf den Dihydronicotinamid-Teil konnte nicht beobachtet werden (Abb. 6).

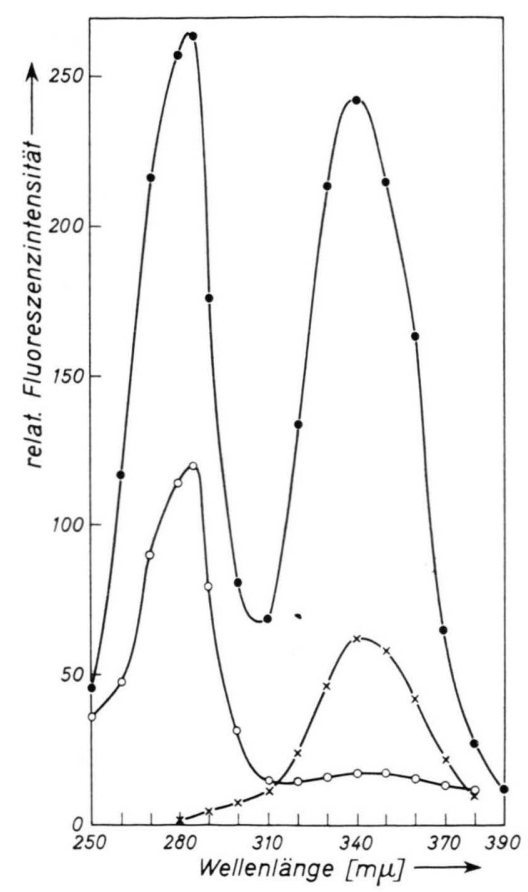

Abb. 6. Fluoreszenzanregungsspektrum: Emission $460 \mathrm{~m} \mu$. $\times--\times$ Dihydronicotinamid-6-mercaptopurin-dinucleotid, $c=$ $1 \cdot 10^{-5} \mathrm{M} ; 0-\mathrm{OLDH}, c=1 \cdot 10^{-5} \mathrm{M} ; 0--\mathrm{LDH}, c=$ $1 \cdot 10^{-5} \mathrm{M}$ und Dihydronicotinamid-6-mercaptopurin-dinucleoid, $c=1 \cdot 10^{-5} \mathrm{M}$; gemessen in $0,2 \mathrm{M}$ Glycin $/ \mathrm{NaOH}-\mathrm{Puffer}$ pH 9,5. Empfängerempfindlichkeit: Stufe 9.

Bei Blockierung der Sulfhydrylreste der LDH durch Quecksilber(II) ionen ${ }^{10}$ beobachteten wir das gleiche Fluoreszenzverhalten des NADH-EnzymKomplexes wie in Gegenwart nativer LDH.

16 K. H. Göbbeler u. C. Woenckhaus, Liebigs Ann. Chem. 700, 180 [1966].

\section{Diskussion}

Für die Wasserstoffdonator-Wirkung des Dihydronicotinamidmononucleotids kann man nur eine sehr schwache Bindung des Coenzymbruchstückes an das Enzym annehmen, denn bei einer MononucleotidKonzentration von $5 \cdot 10^{-4} \mathrm{M}$ konnte weder bei $\mathrm{pH}$ 9,5 noch bei $\mathrm{pH}$ 7,0 in Gegenwart von Pyruvat eine Komplexbildung beobachtet werden. Eine feste Bindung aller Reaktionspartner tritt nicht ein. Eine ähnliche Beobachtung machten wir schon früher bei Verwendung des Coenzymmodells Dihydronicotinamid-pentamethylen-adenosin-diphosphat ${ }^{16}$. Diese Verbindung zeigte keine Komplexbildung, reagierte aber langsam mit einem Überschuß an Substrat mit Dehydrogenasen. Alle Teile des Coenzymmodells, die für die Einlagerung in das "aktive Zentrum“ als Voraussetzung angenommen wurden: Adenosin-, Pyrophosphat- und der Dihydronicotinamidteil, waren vorhanden. Wir schlossen daraus, daß eine Wechselwirkung zwischen den $2^{\prime} \cdot 3^{\prime}$-Hydroxylgruppen des Nicotinamidribosids mit entsprechenden Stellen des Proteins eintritt. Nicotinamid- $2^{\prime} .3^{\prime}$-didesoxyribotid reagierte jedoch im Test mit Alkohol in Gegenwart der Alkohol-Dehydrogenase aus Leber unter Bildung des Dihydronicotinamid-2'. $3^{\prime}$-didesoxyribotids ${ }^{17}$. Für die Bildung eines Coenzym-LDHKomplexes ist die Ribose-diphosphatribose-Brücke zwischen nichtfunktionellem und funktionellem Molekülteil verantwortlich. Beide Coenzymbruchstücke, Adenosin-diphospho-ribose und Dihydronicotinamid-ribose- $5^{\prime}$-diphospho- $\left(\mathrm{P}^{2}\right)-5^{\prime \prime}$-ribose unterdrücken in gleichem Ausmaß die Fluoreszenzemission. Vermutlich wird der symmetrische Pyrophosphorsäureester an das „aktive Zentrum“ angelagert und ändert die Konformation in diesem Teil des Proteins. Dihydronicotinamid-ribose- $5^{\prime}$-diphospho$\left(\mathrm{P}^{2}\right)-5^{\prime \prime}$-ribose bildet einen schwachen Komplex mit der LDH aus. Das Fehlen des Adeninringes in dieser Verbindung drückt sich in der hohen Dissoziationskonstanten aus. Der Ersatz des Adeninringes durch Benzimidazol führt zu einer 20-fachen Vergrößerung der Dissoziationskonstanten gegenüber dem NADH-LDH-Komplex, während beim Dihydronicotinamid-3-desazapurin-dinucleotid nur noch eine 8-fach schwächere Bindung festzustellen ist. Dieses Coenzymmodell erwies sich in den enzymatischen Eigenschaften dem NADH sehr ähnlich ${ }^{14}$. Die An-

17 R. JECK, Diplomarbeit, Frankfurt am Main 1968. 
nahme, daß neben der hydrophoben Bindung des nichtfunktionellen Teils noch eine Wasserstoffbrücke zum N1 bei der Bildung des Coenzym-Enzym-Komplexes eine Bedeutung besitzt, wird durch unsere Untersuchungen unterstützt.

An isolierten Coenzymmodellen wird häufig Energie vom nichtfunktionellen Molekülteil auf den Dihydronicotinamid-Ring übertragen. Man beobachtet dann im Fluoreszenzanregungs-Spektrum Doppelmaxima im Absorptionsbereich beider Coenzymteile. Während beim NADH das Ausbleiben des Effektes mit einer Änderung der Ladungspolarisation durch eine Wasserstoffbrückenbindung an das Enzym erklärt werden kann, ist im Coenzymmodell Dihydronicotinamid-benzimidazol-dinucleotid eine Wasserstoffbrücke an den nichtfunktionellen Molekülteil nicht möglich. Im Dihydronicotinamid-6mercaptopurin-dinucleotid fallen die Absorptionsmaxima des 6-Mercaptopurinteils und des Dihydronicotinamidteils weitgehend zusammen. Im Absorptionsspektrum konnten wir eine Wechselwirkung zwischen beiden Ringsystemen feststellen ${ }^{9}$. Im Komplex mit der LDH fehlt in beiden Fällen eine Intensitätssteigerung der Fluoreszenz bei $460 \mathrm{~m} \mu$ nach Einstrahlen von Licht der Absorptionswellenlängen-

18 J. J. Holbrook, Biochem. Z. 344, 141 [1966].
Maxima. Die fehlende Energieübertragung im Komplex mit dem Enzym weist auf die aufgefaltete Form des Coenzyms nach der Anlagerung an das ,aktive Zentrum “, die schon von VELICK ${ }^{1}$ für den NADHLDH-Komplex gefordert wurde.

Durch Quecksilber(II) ionen wird die katalytische Eigenschaft der LDH reversibel aufgehoben. PfLEIDERER und Mitarbb. ${ }^{10,18}$ beobachteten schon vor einiger Zeit, daß die essentiellen Cysteinreste der LDH nicht an der Coenzym-Enzym-Bindung beteiligt sind. Im Fluoreszenzanregungs- und -emissionsSpektrum konnten wir keinen Unterschied zwischen nativem und gehemmten Enzym feststellen. Die Blockierung der Sulfhydrylreste führt zu keiner Änderung der räumlichen Struktur des „aktiven Zentrums". Beobachtungen von KAPLAN ${ }^{2}$ über das Verhalten der mit $p$-Chloromercuribenzoat blockierten LDH zeigten, daß die charakteristische Enzymfluoreszenz bei Verwendung des Hemmstoffs nicht verändert wird. Für die Verhinderung der NADH. Enzym-Komplexbildung kann nur der große aromatische Rest des Hemmstoffes verantwortlich gemacht werden. Die Zuordnung der essentiellen Cysteinreste der LDH zum „aktiven Zentrum“ des Enzyms konnte bisher nicht geklärt werden.

Dem Verband der Chemischen Industrie danken wir für die Unterstützung der Arbeit. 